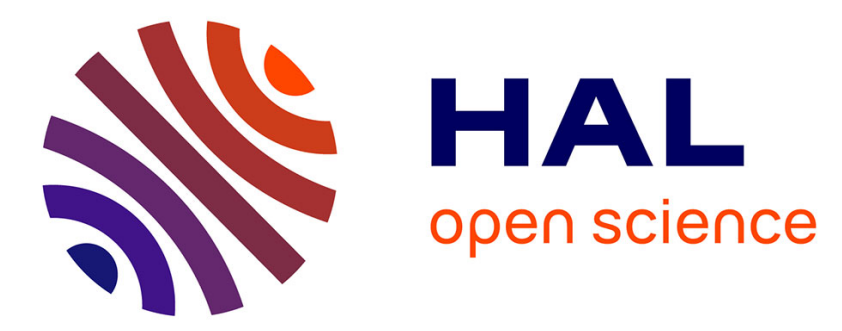

\title{
Solitary transversal waves and vibro-impact motions in infinite chains and rods
}

\author{
Yuri Mikhlin, A.M. Volok
}

\section{To cite this version:}

Yuri Mikhlin, A.M. Volok. Solitary transversal waves and vibro-impact motions in infinite chains and rods. International Journal of Solids and Structures, 2000, 37 (25), pp.3403-3420. 10.1016/S00207683(99)00029-3 . hal-01348408

\section{HAL Id: hal-01348408 \\ https://hal.science/hal-01348408}

Submitted on 23 Jul 2016

HAL is a multi-disciplinary open access archive for the deposit and dissemination of scientific research documents, whether they are published or not. The documents may come from teaching and research institutions in France or abroad, or from public or private research centers.
L'archive ouverte pluridisciplinaire HAL, est destinée au dépôt et à la diffusion de documents scientifiques de niveau recherche, publiés ou non, émanant des établissements d'enseignement et de recherche français ou étrangers, des laboratoires publics ou privés. 


\title{
Solitary transversal waves and vibro-impact motions in infinite chains and rods
}

\author{
Yu V. Mikhlin ${ }^{\mathrm{a}}$, A.M. Volok ${ }^{\mathrm{b}}$ \\ ${ }^{a}$ Department of Applied Mathematics, Kharkov Polytechnical University, 21 Frunze Str. 21, Kharkov 310002, Ukraine \\ ${ }^{\mathrm{b}}$ Department of Applied Mathematics, Dnepropetrovsk State University, Dnepropetrovsk, Ukraine
}

\begin{abstract}
This paper deals with traveling waves in non-linear in ${ }^{\oplus}$ nite elastic systems (chains and rods). A passage to a long wavelength approximation is realized. Conditions of the solitary waves existence are analyzed. The waves with regard to elastic impacts have been investigated.
\end{abstract}

\section{Introduction}

The study of non-linearities due to vibro-impacts is of significant practical importance since they are often encountered in engineering practice. The analysis of the dynamics of strongly non-linear problems requires the development of special analytical techniques suitable for handling strong non-linearities. The problems are analyzed in various publications.

Analytical and numerical studies of vibro-impact oscillations are carried out by Masri and Caughey (1966), by matching linear solutions computed before and after the time instants of impacts. Generally theory of stereomechanical and quasiplastic impacts is presented by Nagaev (1985). Studies of vibroimpact oscillations with analytical/numerical Poincaré maps and geometrical techniques were performed by Shaw and Holmes (1983) and Shaw and Shaw (1989). The applications considered were basically single-DOF systems. Ivanov (1993) studied vibro-impact oscillations introducing auxiliary phase planes and proposed (Ivanov, 1994) a unified approach to the analysis of impact oscillations. Also he studied the problem of multiple impacts (Ivanov, 1995). Zhuravlev (1977) introduced non-smooth spatial transformations of variables to eliminate discontinuities in the equations of motion of the strongly nonlinear vibro-impact systems. The method of non-smooth temporal transformations was developed by 
Pilipchuk (1996). Numerical and experimental investigations of some elastic and rotor-dynamic systems with impact non-linearities were carried out in Emaci et al. (1997). Chaotic motions of vibro-impact systems were studied in works by Shaw and Holmes (1983), Moon et al. (1991), Ivanov (1994), Valkering (1994), Farahanchi and Shaw (1994), Han and Luo (1995). Methods of nonlinear and normal modes theory (Vakakis et al., 1996; Mikhlin, 1996) were used in vibro-impact problems in a work by Mikhlin et al., 1998.

Studies of traveling waves in non-linear elastic systems were carried out by many researchers. Solitary traveling longitudinal waves were analyzed by Ostrovsky and Sutin (1977); the reflection of the soliton at almost fixed and almost free ends were detected in a numerical simulation by Soerensen et al. (1984, 1987) and Clarkson et al. (1986) studied longitudinal wave propagation in a non-linear elastic rod modeled by the non-linear hyperbolic equation, containing odd nonlinearities $\left(u^{3}\right.$ and $\left.u^{5}\right)$ and only one dispersive term with mixed fourth-order derivatives. Samsonov (1995) reduced the initial highly non-linear elastic wave problem governed by coupled PDE to the only one 'double dispersion' equation, describing longitudinal strain waves in a rod; before, he obtained some results in the framework of the $\mathrm{KdV}$ approach (Samsonov, 1984). Solitary traveling waves were analyzed by Toda (1981) in chain systems with special potential. Potapov (1985) determined a principal possibility of the solitary transversal waves existence in essentially non-linear infinite rods.

Although the stability problem is not considered here, some works on the subject will be referred. Zhuravlev (1977) and Vedenova et al. (1985) studied a stability of some vibro-impact motions by employing averaged equations and eliminating a discontinuity in the impact points. Nagaev (1985) considered a stability of vibro-impact motion with a number of inelastic impacts equal to infinity. The author analyzed a one-dimensional system of $n$ solids with masses change by a special exponential rule. Local and global bifurcations of periodic orbits in systems with vibroimpacts and an appearance of chaotic motions were examined in some recent works by Shaw and Holmes (1983), Shaw (1985), Ivanov (1994), Valkering (1994), Farahanchi and Shaw (1994) and Han and Luo (1995).

We analyze here traveling transversal waves in non-linear infinite chains and rods with regards to elastic impacts. The work includes: a passage to a continual, long wavelength approximation in nonlinear infinite chain systems (Section 2), one uses here the translation operator approach; a passage to the long wavelength approximation in non-linear infinite rod system in the framework of Kirchhoff hypothesis (Section 3); an analysis of the solitary waves equations obtained previously (Section 4); an analysis of vibro-impact motions: the solitary waves with impacts in chains (Section 5) and periodical vibro-impact motions (Section 6) are considered.

\section{Non-linear transverse waves in chains}

Consider transverse waves in a symmetric infinite chain consisting of particles (masses of the particles are equal to $m$ ) connected by linear springs (Fig. 1). Stiffnesses of the springs are equal to $\gamma$. The chain is found between two rigid barriers (boundaries) with gaps equal to $L ; a$ is the distance between undisplaced particles.

The renewal coefficients are equal to 1 .

The impact is absolutely elastic. The solitary waves exist only in this case because if the energy fails at the time of impact, the solitary waves collapse.

The displacement vector of the particle with the number $n$ is presented as: 


$$
\bar{S}_{n}=\left\{\begin{array}{l}
\mathbf{W}_{n} \\
\mathbf{V}_{n}
\end{array} \quad n=0, \pm 1, \pm 2, \ldots\right.
$$

where $\mathbf{V}_{n}=a n+\mathbf{U}_{n}$.

Here $\mathbf{W}_{n}$ is a relative transverse displacement, $\mathbf{U}_{n}$ is a relative longitudinal displacement and $\mathbf{V}_{n}$ is an absolute (from zero) longitudinal displacement.

One assumes that the functions continuously depend on $n$ :

$$
\mathbf{W}_{n}=\mathbf{W}(n, t), \quad \mathbf{U}_{n}=\mathbf{U}(n, t) .
$$

Let

$$
a_{n}=\left(\left(\mathbf{W}_{n}-\mathbf{W}_{n-1}\right)^{2}+\left(a+\mathbf{U}_{n}-\mathbf{U}_{n-1}\right)^{2}\right)^{1 / 2}=\left|\bar{S}_{n}-\bar{S}_{n-1}\right| .
$$

The equations governing the transverse motions of this system are expressed as:

$$
\begin{aligned}
& m \mathbf{W}_{n}^{\prime \prime}+\gamma\left(1-a / a_{n}\right)\left(\mathbf{W}_{n}-\mathbf{W}_{n-1}\right)-\gamma\left(1-a / a_{n+1}\right)\left(\mathbf{W}_{n+1}-\mathbf{W}_{n}\right)+P(\mathbf{W})=0 \\
& m \mathbf{U}_{n}^{\prime \prime}+\gamma\left(1-a / a_{n}\right)\left(a+\mathbf{U}_{n}-\mathbf{U}_{n-1}\right)-\gamma\left(1-a / a_{n+1}\right)\left(a+\mathbf{U}_{n+1}-\mathbf{U}_{n}\right)=0 \\
& n=0, \pm 1, \pm 2, \ldots
\end{aligned}
$$

$P(\mathbf{W})$ is the elastic impact function. It is impossible to utilize different forms of the function, for example, power form (Pilipchuk, 1996; Manevitch et al., 1989):

$$
P(X)=C(X / L)^{2 n-1} \quad(C=\text { const., } n \rightarrow \infty) .
$$

It will be restricted to low-frequency (long wavelength) approximation and will be realized in a passage to a continual system in place of system (2).

Consider the function $F(n)$ defined on the integer number set. One writes the Taylor formula for $F(n)$, assuming the function is continuous and infinitely different:

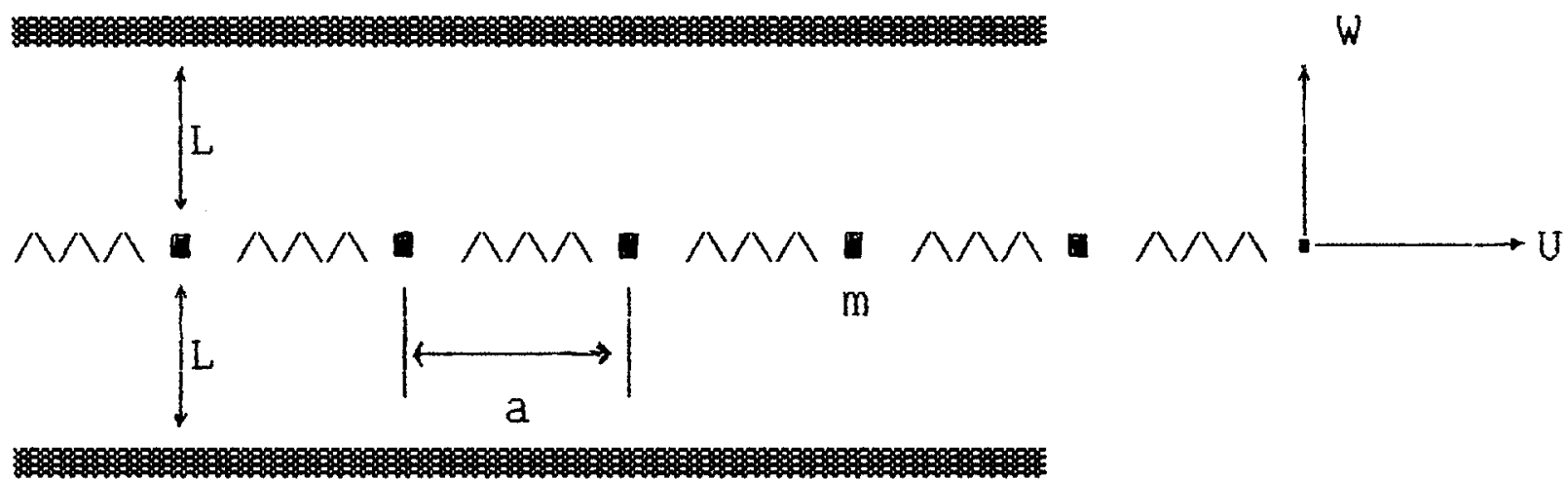

Fig. 1. Symmetric infinite chain consisting of particles connected by linear springs. Masses of the particles are equal to $m$. Stiffnesses of the springs are equal to $\gamma$. The chain is found between two rigid boundaries with gaps equal to $L ; a$ is the distance between undisplaced particles. 


$$
\begin{aligned}
F(n \pm 1) & =F(n)+\frac{\partial}{\partial n}(F(n))( \pm 1)+\frac{\partial^{2}}{\partial n^{2}}(F(n))( \pm 1)^{2} / 2 !+\cdots \\
& =\left(1+( \pm 1) \frac{\partial}{\partial n}+\frac{( \pm 1)^{2}}{2 !} \frac{\partial^{2}}{\partial n^{2}}+\cdots\right) F(n)=\mathrm{e}^{ \pm \partial / \partial n} F(n),
\end{aligned}
$$

utilising an operator form of the notation.

The operator (3) is used by Pilipchuk (1996) and Manevitch et al. (1989), and called 'a translation operator'. There are obvious combinations:

$$
\begin{aligned}
& \left.F(n)-F(n-1)=\left(1-\mathrm{e}^{-\partial / \partial n}\right) F(n)=\sum_{k=1}^{\infty} \frac{(-1)^{k+1}}{k !} \frac{\partial^{k}}{\partial n^{k}}\right) F(n) \\
& \left.F(n+1)-F(n)=\left(\mathrm{e}^{\partial / \partial n}-1\right) F(n)=\sum_{k=1}^{\infty} \frac{1}{k !} \frac{\partial^{k}}{\partial n^{k}}\right) F(n) .
\end{aligned}
$$

Introduce the operators:

$$
A=1-\mathrm{e}^{-\partial / \partial n} ; \quad B=\mathrm{e}^{\partial / \partial n}-1 .
$$

One passes to a continual system replacing the discrete variable $n$ by a continuous variable $x$.

Consider traveling stationary waves. Corresponding solutions are described by functions of a single argument named 'phase',

$$
\Phi=k x-\omega t,
$$

where: $x$ is the space coordinate; $k$ is the wavenumber, $k=2 \pi / \lambda ; \lambda$ is the length of the wave; $\omega$ is the frequency of the traveling wave.

Let $\mathbf{W}=\mathbf{W}(\Phi), \mathbf{U}=\mathbf{U}(\Phi), \mathbf{V}=\mathbf{V}(\Phi)$, respectively,

$$
\frac{\partial}{\partial n}=(k a) \frac{\mathrm{d}}{\mathrm{d} \Phi}, \quad \frac{\partial}{\partial t}=-\omega \frac{\mathrm{d}}{\mathrm{d} \Phi} ; \quad \mathbf{V}(\Phi)=n a+\mathbf{U}(\Phi) \quad \text { and } \quad \partial \mathbf{V} / \partial n=a+\partial \mathbf{U} / \partial n, \text { etc. }
$$

Using the formulas, one obtains new representations for the operators $A, B$ from (5):

$$
\begin{aligned}
& A=\sum_{r=1}^{\infty} \frac{(-1)^{r+1}}{r !}(a k)^{r} \frac{\partial^{r}}{\partial \Phi^{r}} \\
& B=\sum_{r=1}^{\infty} \frac{1}{r !}(a k)^{r} \frac{\partial^{r}}{\partial \Phi^{r}} \quad(k a \ll 1, \text { this is a long wavelength approximation). }
\end{aligned}
$$

Note that a passage to a continual system of high-frequency approximation is possible using a continualization of an envelope (Kosevich and Kovalev, 1975; Manevitch et al., 1989):

$$
\mathbf{U}_{n} \rightarrow(-1)^{n} \mathbf{U}_{n} .
$$

Taking into account (6) and (7) we obtain a system of ODEs characterizing the traveling stationary waves, in place of system (2): 


$$
\begin{aligned}
& \omega^{2} \mathbf{W}^{\prime \prime}+v^{2}\left[\left(1-\frac{a}{\sqrt{A(\mathbf{W})^{2}+A(\mathbf{V})^{2}}}\right) A(\mathbf{W})-\left(1-\frac{a}{\sqrt{B(\mathbf{W})^{2}+B(\mathbf{V})^{2}}}\right) B(\mathbf{W})\right]+P(\mathbf{W})=0 \\
& \left.\omega^{2} \mathbf{V}^{\prime \prime}+v^{2}\left[\left(1-\frac{a}{\sqrt{A(\mathbf{W})^{2}+A(\mathbf{V})^{2}}}\right) A(\mathbf{V})-1-\frac{a}{\sqrt{B(\mathbf{W})^{2}+B(\mathbf{V})^{2}}}\right) B(\mathbf{V})\right]=0
\end{aligned}
$$

where $v^{2}=\gamma / m$; here the prime means a derivation by $\Phi$.

One introduces a small parameter $\mu=k^{2}$, also let $\theta=a^{2}$.

One expands the components of system (8) in the Taylor series in the vicinity of $\mu=0$ and retains the leading terms. Note that

$$
\mathbf{V}^{\prime}=\mathrm{d} \mathbf{V} / \mathrm{d} \Phi=\frac{\mathrm{d}}{\mathrm{d} \Phi}(a n+\mathbf{U}(\Phi))=\mathbf{U}^{\prime}+\frac{\mathrm{d}(a n)}{\mathrm{d}(a n k-\omega t)}=\frac{1}{k}+\mathbf{U}^{\prime} .
$$

Finally, making a transformation,

$$
\mathbf{U}(\Phi) \rightarrow k \delta \mathbf{U}(\Phi), \quad \mathbf{W}(\Phi) \rightarrow \epsilon \mathbf{W}(\Phi)
$$

(amplitudes $\varepsilon, \delta$ etc. $\epsilon, \delta>0$ ) in order that $\left|\mathbf{U}^{\prime}(\Phi)\right| \leqslant 1,\left|\mathbf{W}^{\prime}(\Phi)\right| \leqslant 1$, one obtains after some transformations of the following system:

$$
\begin{aligned}
& {\left[\omega^{2}-\theta v^{2} \mu^{2}\left(\delta \mathbf{U}^{\prime}+\epsilon^{2} \mathbf{W}^{\prime 2} / 2\right)\right] \epsilon \mathbf{W}^{\prime \prime}-\theta v^{2} \mu^{2}\left(\epsilon^{2} \mathbf{W}^{\prime} \mathbf{W}^{\prime \prime}+\delta \mathbf{U}^{\prime \prime}\right) \epsilon \mathbf{W}^{\prime}+O\left(\mu^{3}\right)=0} \\
& {\left[\omega^{2}-\theta v^{2} \mu^{2}\left(\delta \mathbf{U}^{\prime}+\epsilon^{2} \mathbf{W}^{\prime 2} / 2\right)\right] \delta \mathbf{U}^{\prime \prime}-\theta v^{2}\left[\mu\left(\epsilon^{2} \mathbf{W}^{\prime} \mathbf{W}^{\prime \prime}+\delta \mathbf{U}^{\prime \prime}\right)+\mu^{2} \delta \mathbf{U}^{\prime}\left(\epsilon^{2} \mathbf{W}^{\prime} \mathbf{W}^{\prime \prime}+2 \delta \mathbf{U}^{\prime \prime}\right)\right.} \\
& \left.\quad+\frac{\theta}{12} \mu^{2}\left(\delta \mathbf{U}^{\prime \prime \prime \prime}+\epsilon^{2} \mathbf{W}^{\prime} \mathbf{W}^{\prime \prime \prime \prime}\right)-3 \mu^{2}\left(\epsilon^{2} \mathbf{W}^{\prime} \mathbf{W}^{\prime \prime}+\delta \mathbf{U}^{\prime \prime}\right)\left(\delta \mathbf{U}^{\prime}+\epsilon^{2} \mathbf{W}^{\prime 2} / 2\right)\right]+O\left(\mu^{3}\right)=0 .
\end{aligned}
$$

The impact function is not presented in (10) and will be used later. In new variables the impact happens if $|\mathbf{W}|=L / \epsilon$.

One obtains from the first equation of (10) the estimate: $\omega^{2}=O\left(\mu^{2}\right)$.

Let $\omega^{2}=\omega_{0}^{2}+O\left(\mu^{3}\right)$.

From the second equation of (10), retaining the leading terms of $O(\mu)$, we have the following equation:

$$
\delta \mathbf{U}^{\prime \prime}+\epsilon^{2} \mathbf{W}^{\prime} \mathbf{W}^{\prime \prime}=0 .
$$

Integrating by $\Phi$, one has $\delta \mathbf{U}^{\prime}+\epsilon^{2} \mathbf{W}^{\prime 2} / 2=\mathscr{D}$ ( $\mathscr{D}$ being arbitrary constant).

Write out the first equation of (10) taking into account (11) and the terms of $O\left(\mu^{2}\right)$ in the second equation in (10):

$$
\begin{aligned}
& {\left[\omega_{0}^{2}-\theta \boldsymbol{v}^{2} \mathscr{D}\right] \epsilon \mathbf{W}^{\prime \prime}=0} \\
& {\left[\omega_{0}^{2}-\theta \boldsymbol{v}^{2} \mathscr{D}\right] \epsilon^{2} \mathbf{W}^{\prime} \mathbf{W}^{\prime \prime}-\theta \boldsymbol{v}^{2}\left[\delta \mathbf{U}^{\prime} \epsilon^{2} \mathbf{W}^{\prime} \mathbf{W}^{\prime \prime}-\frac{\theta}{12}\left(\delta \mathbf{U}^{\prime \prime \prime \prime}+\epsilon^{2} \mathbf{W}^{\prime} \mathbf{W}^{\prime \prime \prime \prime}\right)\right]=0 .}
\end{aligned}
$$

In the case $\mathbf{W}^{\prime \prime}=0$ one has, with regard to impacts (in a position $|\mathbf{W}|=L / \epsilon$ ), a non-smooth 'saw-tooth' solution (Pilipchuk, 1996). Consider here other solutions. Let $\omega_{0}^{2}-\theta v^{2} \mathscr{D}=0$. It is clear that $\mathscr{D} \geq 0$.

The second equation of (12) gives us the following: 


$$
\mathbf{W}^{\prime \prime \prime}+b_{1} \mathbf{W}^{\prime}-b_{3} \mathbf{W}^{\prime 3}=0
$$

where $b_{1}=4 \mathscr{D} / \theta, b_{3}=2 \epsilon^{2} / \theta$.

The analysis of solitary waves in elastic chains will be carried out in Section 4; the impact function will be considered in Section 5.

\section{Non-linear transverse waves in rods}

Consider the infinite elastic rod being between two ideal and absolutely fixed catches (barriers) with gaps equal to $L$.

The impact process is not considered in this section and will be considered later.

One describes the plane rod dynamics using equations of coupled longitudinal-bending (transverse) vibrations of rods, obtained by Potapov (1985). Note that the following analysis will be made for nonlinear rods within the framework of the Kirchhoff hypothesis (Kauderer, 1958).

The equations may be deduced from expressions of potential and kinetic energies containing quadratic, cubic terms and principal terms of the fourth degree.

Corresponding equations of motion are expressed as (Potapov, 1985):

$$
\begin{aligned}
\mathbf{U}_{t t}- & C_{S}^{2}\left(1+6 \alpha_{1} / E \mathbf{U}_{X}\right) \mathbf{U}_{X X}-v^{2} R_{\rho}^{2} \frac{\partial^{2}}{\partial X^{2}}\left[\mathbf{U}_{t t}-C_{\tau}^{2} \mathbf{U}_{X X}\right]=\frac{\partial}{\partial X}\left[\left(C_{S}^{2} / 2+C_{M}^{2}\right) \mathbf{W}_{X}^{2}\right. \\
& \left.+R_{Y}^{2} \mathbf{W}_{X t}^{2}+R_{Y}^{2} \alpha_{2} / \rho_{0} \mathbf{W}_{X X}^{2}\right]-R_{Y}^{2} \frac{\partial^{2}}{\partial X \partial t}\left[\mathbf{W}_{X} \mathbf{W}_{X t}\right]+\frac{\partial^{2}}{\partial X^{2}}\left[\left(C_{S}^{2}+4 C_{\tau}^{2}\right) R_{Y}^{2} \mathbf{W}_{X} \mathbf{W}_{X X}\right] \\
& \mathbf{W}_{t t}+\left(C_{S}^{2} R_{Y}^{2}+v^{2} C_{\tau}^{2} R_{Z}^{2}\right) \mathbf{W}_{X X X X}+v^{2} \mathscr{I}_{2} / F \frac{\partial^{4}}{\partial X^{4}}\left[\mathbf{W}_{t t}-C_{\tau}^{2} \mathbf{W}_{X X}\right]-R_{Y}^{2}(1-v) \mathbf{W}_{X X t t} \\
= & \frac{\partial}{\partial X}\left[R_{Y}^{2} \mathbf{U}_{X t} \mathbf{W}_{X t}+\left(C_{S}^{2}+2 C_{M}^{2}\right) \mathbf{U}_{X} \mathbf{W}_{X}+\left(C_{S}^{2}+4 C_{\tau}^{2}\right) R_{Y}^{2} \mathbf{U}_{X X} \mathbf{W}_{X X}+\frac{1}{2} C_{S}^{2} \mathbf{W}_{X}^{3}\right] \\
& +R_{Y}^{2} \frac{\partial^{2}}{\partial X^{2}}\left[\left(C_{S}^{2}+4 C_{\tau}^{2}\right) \mathbf{U}_{X X} \mathbf{W}_{X}+2 \alpha_{2} / \rho_{0} \mathbf{U}_{X} \mathbf{W}_{X X}\right]_{y}-R_{Y}^{2} \frac{\partial^{2}}{\partial X \partial t}\left[2 \mathbf{U}_{X} \mathbf{W}_{X t}+\mathbf{W}_{X} \mathbf{U}_{X t}\right] .
\end{aligned}
$$

Here: $\mathbf{U}(X, t)$ characterizes a longitudinal displacement; $\mathbf{W}(X, t)$ characterizes a transverse displacement of a middle curve of the rod; the coefficients $\alpha_{1}=(E / 2)+\frac{3}{2} \lambda+\left(\frac{1}{6}-v\right) v_{1}+(1-2 v) v_{2}+\frac{4}{3} v_{3}$ and $\alpha_{2}=$ $(E / 2)+\frac{1}{2} v_{1}+3 v_{2}+4 v_{3}$ determine geometrical and physical non-linearities; $C_{S}=\sqrt{E / \rho_{0}}$ is the velocity of longitudinal waves propagation in the $\operatorname{rod} ; C_{\tau}=\sqrt{M / \rho_{o}}$ is the velocity of translation waves propagation in the rod; $C_{M}=\sqrt{\lambda / \rho_{0}} ; \rho_{0}$ is the density per unit length; $E$ is the elasticity modulus of the material; $v$ is Poisson's coefficient; $\lambda$ and $M$ are Lamé constants of the second-order; $v_{1}, v_{2}, v_{3}$ are one's of the third-order; $\mathscr{I}_{\rho}=\iint_{F}\left(Y^{2}+Z^{2}\right) \mathrm{d} F$ is the polar moment of inertia; $\mathscr{I}_{Y}=\iint_{F} Y^{2} \mathrm{~d} F, \mathscr{I}_{Z}=\iint_{F} Z^{2} \mathrm{~d} F$ are the axial moments of inertia; $\mathscr{I}_{1}=\iint_{F} Z^{4} \mathrm{~d} F, \mathscr{I}_{2}=\iint_{F} Z^{2}\left(Y^{2}+Z^{2} / 2\right) \mathrm{d} F$ are the geometrical moments of inertia of the fourth-order; $R_{\rho}=\sqrt{\mathscr{I}_{\rho} / F}$ is the polar radius of inertia; and $R_{Y, Z}=\sqrt{\mathscr{I}_{Y, Z} / F}$ are the axial radii of inertia.

Consider, just as previously, traveling stationary waves of the single argument ('phase'): $\mathbf{U}=\mathbf{U}(\Phi)$, $\mathbf{W}=\mathbf{W}(\Phi)$, where $\Phi=k x-\omega t$.

One transforms to dimensionless variables:

$$
\mathbf{U}(\Phi) \rightarrow \epsilon_{1} \mathbf{U}(\Phi), \quad \mathbf{W}(\Phi) \rightarrow \epsilon_{2} \mathbf{W}(\Phi)
$$

in order that initial values of the new variables 


$$
\left|\mathbf{U}^{\prime}(0)\right|=1, \quad\left|\mathbf{W}^{\prime}(0)\right|=1,
$$

where $\left({ }^{\prime}\right)=\mathrm{d} / \mathrm{d} \Phi, \epsilon_{1}, \epsilon_{2}>0$.

We are restricted to a long wavelength approximation corresponding to the Kirchhoff hypothesis. Orders of the principal parameters of wave processes are as follows:

$$
\begin{aligned}
& k^{2}=\mu \ll 1, \quad \omega=O\left(k^{2}\right) \\
& \epsilon_{1}=k \delta, \quad \delta=O(1) ; \quad \epsilon_{2}=\epsilon, \quad \epsilon=O(1) .
\end{aligned}
$$

Eqs. (14) can be assumed to be of the form of ODEs:

$$
\begin{gathered}
k \delta \omega^{2} \mathbf{U}^{\prime \prime}-C_{S}^{2}\left(1+\frac{6}{E} \alpha_{1}^{2} \delta k \mathbf{U}^{\prime}\right) \delta k^{3} \mathbf{U}^{\prime \prime}-v^{2} R_{\rho}^{2} \delta k^{3}\left(\omega^{2}-C_{\tau}^{2} k^{2}\right) \mathbf{U}^{(4)}=k \epsilon^{2}\left[\left(C_{S}^{2} / 2+C_{M}^{2}\right) k^{2} \mathbf{W}^{\prime 2}\right. \\
\left.+R_{Y}^{2} k^{2} \omega^{2} \mathbf{W}^{\prime \prime}{ }^{2}+R_{Y}^{2} \alpha_{2} / \rho_{0} k^{4} \mathbf{W}^{\prime \prime}\right]^{\prime}-R_{Y}^{2} \omega^{2} \epsilon^{2} k^{3}\left[\mathbf{W}^{\prime} \mathbf{W}^{\prime \prime}\right]^{\prime \prime}+\left(C_{S}^{2}+4 C_{\tau}^{2}\right) R_{Y}^{2} k^{5} \epsilon^{2}\left[\mathbf{W}^{\prime} \mathbf{W}^{\prime \prime}\right]^{\prime \prime} ; \\
\epsilon \omega^{2} \mathbf{W}^{\prime \prime}+\left(C_{S}^{2} R_{Y}^{2}+v^{2} C_{\tau}^{2} R_{Z}^{2}\right) \epsilon k^{4} \mathbf{W}^{(4)}+v^{2} \mathscr{I}_{2} k^{4} \epsilon / F\left(\omega^{2}-C_{\tau}^{2} k^{2}\right) \mathbf{W}^{(6)}-R_{Y}^{2}(1-v) \epsilon \omega^{2} k^{2} \mathbf{W}^{(4)} \\
=k\left[R_{Y}^{2} \epsilon \delta \omega^{2} k^{3} \mathbf{U}^{\prime \prime} \mathbf{W}^{\prime \prime}+\left(C_{S}^{2}+2 C_{M}^{2}\right) \epsilon \delta k^{3} \mathbf{U}^{\prime} \mathbf{W}^{\prime}+\left(C_{S}^{2}+4 C_{\tau}^{2}\right) R_{Y}^{2} \epsilon \delta k^{5} \mathbf{U}^{\prime \prime} \mathbf{W}^{\prime \prime}+\frac{1}{2} C_{S}^{2} \epsilon^{3} k^{3} \mathbf{W}^{\prime}\right]^{\prime} \\
-R_{Y}^{2} k^{4} \omega^{2} \epsilon \delta\left[2 \mathbf{U}^{\prime} \mathbf{W}^{\prime \prime}+\mathbf{W}^{\prime} \mathbf{U}^{\prime \prime}\right]^{\prime \prime}+R_{Y}^{2} \epsilon \delta k^{5}\left[\left(C_{S}^{2}+4 C_{\tau}^{2}\right) \mathbf{U}^{\prime \prime} \mathbf{W}^{\prime}+2 \alpha_{2} / \rho_{0} \mathbf{U}^{\prime} \mathbf{W}^{\prime \prime}\right]^{\prime \prime} .
\end{gathered}
$$

Then one can reduce the order of the system integrating this. Utilize the expansion

$$
\omega^{2}=\mu^{2} \omega_{0}^{2}+\mu^{3} \omega_{1}^{2}+O\left(\mu^{4}\right)
$$

Preserving the leading terms by the small parameter $\mu$, one obtains from the first equation of (19) after integration, the following:

$$
\delta \mathbf{U}^{\prime}=-\frac{\epsilon^{2}}{2}\left(1+2\left(C_{M} / C_{S}\right)^{2}\right) \mathbf{W}^{\prime 2}+\mathscr{D}_{1},
$$

$\mathscr{D}_{1}$ is an arbitrary constant.

By substituting the expression (19) to the second equation of (17) after integration, one has

$$
\mathbf{W}^{\prime \prime \prime}+p_{1} \mathbf{W}^{\prime}+p_{3} \mathbf{W}^{\prime 3}=\mathscr{D}_{2}
$$

where

$$
\begin{aligned}
& p_{1}=\left(\omega_{0}^{2}-\left(C_{S}^{2}+2 C_{M}^{2}\right) \mathscr{D}_{1}\right) /\left(C_{S}^{2} R_{Y}^{2}+v^{2} C_{\tau}^{2} R_{Z}^{2}\right) \\
& p_{3}=-\frac{1}{2} C_{S}^{2} \epsilon^{2}\left(1-\left(1+2\left(C_{M} / C_{S}\right)^{2}\right)^{2}\right) /\left(C_{S}^{2} R_{Y}^{2}+v^{2} C_{\tau}^{2} R_{Z}^{2}\right)
\end{aligned}
$$

$\mathscr{D}_{2}$ is an arbitrary constant.

In the case of the solitary transversal waves $\mathscr{D}_{2}=0$.

Note that the solitary transversal waves exist if $p_{1}<0$, that is $\left(C_{S}^{2}+2 C_{M}^{2}\right) \mathscr{D}_{1}>\omega_{0}^{2}$.

The last equation of (20) is similar to Eq. (13). But the phase places of Eqs. (13) and (20) are different. The point is that a shear is absent in the chain system under consideration. 


\section{Solitary waves in rods and chains}

Eq. (2) in the case $\mathscr{D}_{2}=0$ [or Eq. (13)] is well studied, and here it will be selected conditions of the solitary waves existence. Four principal situations are possible:
(a) $p_{1}<0, \quad p_{3}<0$;
(b) $p_{1}>0, \quad p_{3}>0$;
(c) $p_{1}<0, \quad p_{3}>0$
(d) $p_{1}>0, \quad p_{3}<0$.

The corresponding phase portraits are presented in Fig. 2. Every diagram has its own scale by axes. Curves on the places conform to the first integral of Eqs. (20) or (13):

$$
\mathbf{W}^{\prime \prime 2}+p_{1} \mathbf{W}^{\prime 2}+p_{3} \mathbf{W}^{\prime 4} / 2=H
$$

( $H$ is an arbitrary constant, this is an energy of the system).

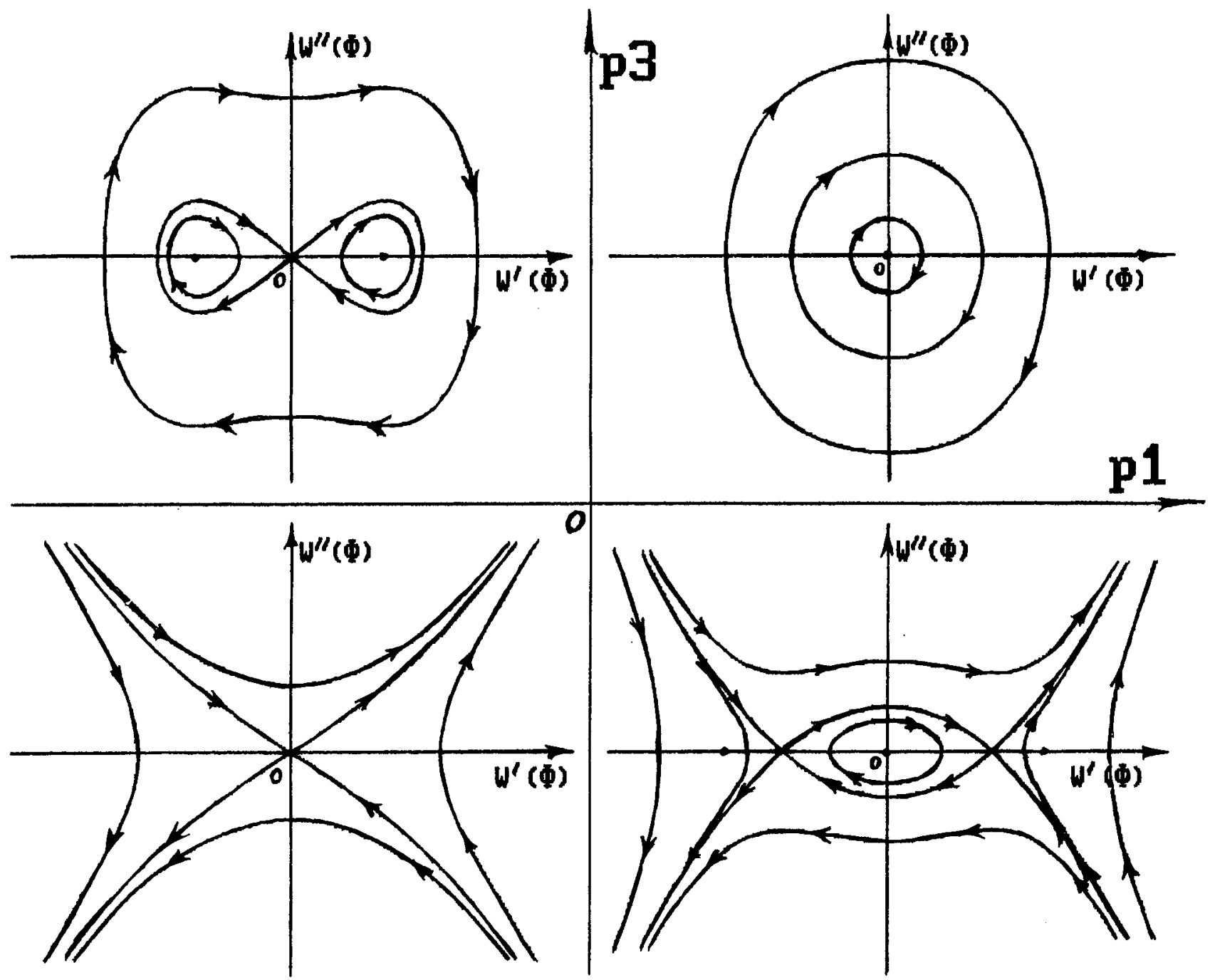

Fig. 2. Phase portraits (W', $\left.\mathbf{W}^{\prime \prime}\right)$ of Eq. (20) [or (13)] Cases (a)-(d). Every diagram has its own scale by axes. Curves on the places conform to the first integral (23) of Eq. (20) or (13). Case (a): the solitary waves are missing; Case (b): the solitary waves are missing; Case (c): the separatrix $(H=0)$ corresponds to the solitary traveling wave; Case $(\mathrm{d})$ : the separatrix $\left(H=q, q=b_{1}^{2} /\left(2 b_{3}\right)\right)$ corresponds to the solitary traveling wave. 
Further, let $b_{1}=\left|p_{1}\right|, b_{3}=\left|p_{3}\right|$.

Case (a) The separatrix $(H=0)$ divides unperiodical solutions, and the solitary waves are missing.

Case (b) All solutions are periodic, and the separatrix are missing.

Case (c) The separatrix $(H=0)$ corresponds to the solitary traveling wave.

Case (d) The separatrix $\left(H=q\right.$, where $\left.q=b_{1}^{2} /\left(2 b_{3}\right)\right)$ confines a region of a periodical solution enclosing the equilibrium position $(0,0)$. The separatrix corresponds to the solitary traveling wave.

\subsection{Consider Case (c) appropriate to the case of the solitary waves in a non-linear rod system}

It is possible to write out the well-known solution corresponding to the separatrix:

$$
\mathbf{W}^{\prime}= \pm \frac{1}{\sqrt{q} \operatorname{ch} \Psi}
$$

where $q=b_{3} /\left(2 b_{1}\right), \Psi=\mathbf{A} \pm \sqrt{b_{1}} \Phi$, and the arbitrary parameter $\mathbf{A}$ (this is equal to the initial phase by a traveling variable) may be eliminated without loss of generality by imposing the initial condition.

Integrating the relation (24) one obtains

$$
\mathbf{W}=\mathbf{B} \pm \sqrt{2 / b_{3}} \operatorname{arctg} \operatorname{sh}\left(\sqrt{b_{1}} \Phi\right),
$$

B being an arbitrary constant. In addition, one writes:

$$
\left.\mathbf{W}^{\prime \prime}= \pm \quad-\sqrt{2 b_{1}^{2} / b_{3}} \frac{\operatorname{th}\left(\sqrt{b_{1}} \Phi\right)}{\operatorname{ch}\left(\sqrt{b_{1}} \Phi\right)}\right) .
$$

The solution $\mathbf{W}(\Phi)$ just $\mathbf{W}^{\prime}(\Phi)$ and $\mathbf{W}^{\prime \prime}(\Phi)$ are bounded functions.

Taking advantage of Eq. (19) one has the function $\mathbf{U}(\Phi)$ :

$$
\begin{aligned}
& \delta \mathbf{U}^{\prime}=\mathscr{D}-\epsilon^{2}\left(\frac{1}{2}+\left(C_{M} / C_{S}\right)^{2}\right) \mathbf{W}^{\prime 2} \\
& \mathbf{U}=\mathbf{C}+\mathscr{D} \Phi / \delta-\frac{\epsilon^{2}}{\delta} \sqrt{b_{1}} / b_{3}\left(\frac{1}{2}+\left(C_{M} / C_{S}\right)^{2}\right) \operatorname{th}\left(\sqrt{b_{1}} \Phi\right) .
\end{aligned}
$$

Here $\mathbf{C}$ is an arbitrary constant.

The constant $\mathscr{D}$ has the meaning of the preliminary deformation of the rod. The function $\mathbf{U}(\Phi)$ is unbounded in this case. The constant $\mathscr{D}$ is not equal to zero because in the opposite case $(\mathscr{D}=0)$ one has $\omega_{0}^{2}<0$.

\subsection{Consider Case (d) appropriate to the non-linear chain system}

It is possible to write out the well-known solution corresponding to the separatrix:

$$
\mathbf{W}^{\prime}= \pm \sqrt{b_{1} / b_{3}} \operatorname{th}\left(\sqrt{b_{1} / 2} \Phi\right)
$$

(the initial phase A may be eliminated without loss of generality by imposing the initial condition); one obtains, integrating (27):

$$
\mathbf{W}=\mathbf{B} \pm \sqrt{2 / b_{3}} \ln \operatorname{ch}\left(\sqrt{b_{1} / 2} \Phi\right)
$$


also

$$
\mathbf{W}^{\prime \prime}= \pm \sqrt{b_{1}^{2} /\left(2 b_{3}\right)} \frac{1}{\operatorname{ch}^{2}\left(\sqrt{b_{1} / 2} \Phi\right)}
$$

where $\mathbf{B}$ is an arbitrary constant.

The transformation to new variables (9) was introduced provided that $\left|\mathbf{U}^{\prime}(\Phi)\right| \leq 1,\left|\mathbf{W}^{\prime}(\Phi)\right| \leq 1$. One obtains from (27) that

$$
\left|\mathbf{W}^{\prime}(\Phi)\right| \leq \sqrt{b_{1} / b_{3}}, \quad \text { hence } b_{1}=b_{3} \quad \text { or } 4 \mathscr{D} / \theta=2 \epsilon^{2} / \theta \quad \text { that is } \mathscr{D}=\epsilon^{2} / 2 \text {. }
$$

One has from here that

$$
\omega_{0}^{2}=\theta v^{2} \epsilon^{2} / 2
$$

This is a relation between the wave frequency $\omega$ and the wave amplitude $\epsilon$.

Using Eq. (20) one has:

$$
\delta \mathbf{U}^{\prime}=\epsilon^{2} / 2\left(1-\mathbf{W}^{\prime 2}\right)=\frac{\epsilon^{2}}{\operatorname{ch}^{2}\left(\sqrt{b_{1} / 2} \Phi\right)}
$$

and

$$
\mathbf{U}=\mathbf{C}+\frac{\epsilon^{2}}{2 \delta} \sqrt{2 / b} \operatorname{th}\left(\sqrt{b_{1} / 2} \Phi\right)
$$

C is an arbitrary constant.

The condition $\left|\mathbf{U}^{\prime}(\Phi)\right| \leq 1$ gives us the relation:

$$
\delta=\frac{\epsilon^{2}}{2} .
$$

Formula (32) has the following meaning: maximal longitudinal and transverse deformations are connected.

One also obtains

$$
b_{1} / 2=4 \mathscr{D} / \theta=(\epsilon / a)^{2} .
$$

Let $V=1 / a$. We now extract the expressions of the solutions which describe the solitary waves in a non-linear chain system:

$$
\begin{aligned}
& \mathbf{W}=\mathbf{B} \pm \frac{1}{V \epsilon} \ln \operatorname{ch}(V \epsilon \Phi) \\
& \mathbf{W}^{\prime}=\operatorname{th}(V \epsilon \Phi) \\
& \mathbf{W}^{\prime \prime}=\frac{ \pm V \epsilon}{\operatorname{ch}^{2}(V \epsilon \Phi)}
\end{aligned}
$$

Plots of the functions (34) are presented in Fig. 3. The diagrams have different scales by vertical axis. The argument $\Phi \epsilon[-4,4]$. 


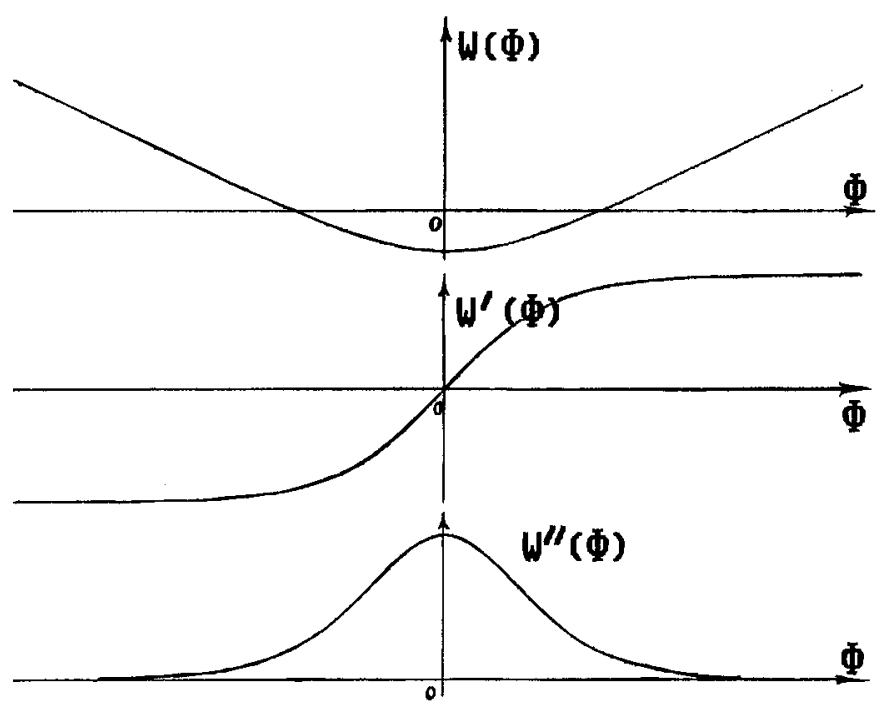

Fig. 3. Plots of the solitary waves (34), Case (d). Diagrams have different scales by vertical axis. The argument $\Phi \epsilon[-4,4]$.

$$
\begin{aligned}
\mathbf{U} & =\mathbf{C}+\frac{1}{V \epsilon} \operatorname{th}(V \epsilon \Phi) \\
\mathbf{U}^{\prime} & =\frac{1}{\operatorname{ch}^{2}(V \epsilon \Phi)} .
\end{aligned}
$$

As a consequence one has the equality: $\mathbf{U}^{\prime}=1-\mathbf{W}^{\prime 2}$.

Here the constant $\mathbf{B}$ defines some initial value of the transverse displacement, the constant $\mathbf{C}$ defines some initial value of the longitudinal displacement.

The expressions (34) and (35) represent a one-parameter family of solutions. The amplitude $\epsilon$ is a free parameter.

\section{Vibro-impact motions in chains}

The solution $\mathbf{W}(\Phi)$ in a form of the solitary wave (34) is unbounded at infinity. In the presence of barriers the impacts take place necessarily.

Construct the phase place $\left(\mathbf{W}, \mathbf{W}^{\prime}\right)$ of the obtained solution. At the time of impact a sign of the velocity changes and the following principal cases are possible:

1. The impacts are possible and there is a 'jumping' to the same phase trajectory.

2. The impacts are possible and there is a 'jumping' to another phase trajectory.

3. The impacts are impossible, that is the solution does not exist in the presence of barriers.

In the new coordinates [see the transformation (9)] the distance between the barriers equal to $2 L / \epsilon$.

Using the expression (34), Case (d), one obtains the relationship $\mathbf{W}^{\prime}(\mathbf{W})$ :

$$
\mathbf{W}^{\prime}= \pm \operatorname{sign}(\Phi) \sqrt{1-\mathrm{e}^{ \pm 2 V \epsilon(\mathbf{B}-\mathbf{W})}},
$$

where $V^{2}=b_{1} /\left(2 \epsilon^{2}\right)$.

Branch ' + ' (branch $\alpha$ ) of the solution corresponds to $\mathbf{W}>\mathbf{B}$, and one '-' (branch $\beta$ ) corresponds to $\mathbf{W}<\mathbf{B}$. 
The phase place $\left(\mathbf{W}, \mathbf{W}^{\prime}\right)$ is depicted in Fig. 4. The solutions increase or decrease indefinitely. In Fig. 4 the constant $\mathbf{B}=-1 ;-1<\mathbf{W}<4$ (branch $\alpha$ ), $-4<\mathbf{W}<-1$ (branch $\beta$ ).

If barriers exist, three variants are possible:

1. $-L / \epsilon<\mathbf{B}<L / \epsilon$.

2. $\mathbf{B}<-L / \epsilon$ or $\mathbf{B}>L / \epsilon$.

3. $\mathbf{B}= \pm L / \epsilon$.

\subsection{Consider the variant 1}

The corresponding phase portrait is presented in Fig. 5. Here $\mathbf{B}=-1, L / \epsilon=1.7$, the range of values of the variable $\mathbf{W}$ is the same as in Fig. 4.

There are two separated modes of vibrations, each of them strikes its own barrier. It reaches a 'jumping' to the same vibration mode (Fig. 5).

Evaluate the period of the vibro-impact modes. One uses the representation (34):

$$
\mathbf{W}=\mathbf{B}+\frac{1}{V \epsilon} \ln \operatorname{ch}(V \epsilon \Phi) .
$$

The impact takes place if $\mathbf{W}=L / \epsilon($ branch $\alpha)$. One has from here

$$
\begin{aligned}
& L / \epsilon=\mathbf{B}+\frac{1}{V \epsilon} \ln \operatorname{ch}(V \epsilon \Phi) . \\
& \Phi=\frac{1}{V \epsilon} \ln \left(\mathrm{e}^{(L-\mathbf{B} \epsilon) V} \pm \sqrt{\mathrm{e}^{2(L-\mathbf{B} \epsilon) V}-1}\right) .
\end{aligned}
$$

In Fig. 5 a point $A_{1}$ corresponds to the sign ' - ' in relation (37) (here $\Phi=\Phi_{1}$ ), a point $A_{2}$ corresponds to the sign ' + ' (here $\Phi=\Phi_{2}$ ), moreover $\Phi_{1}=-\Phi_{2}$.

One assumes that the impact is stereomechanical and takes place instantly. The stereomechanical impact theory was established by Newton (Goldsmith, 1960; Nagaev, 1985).

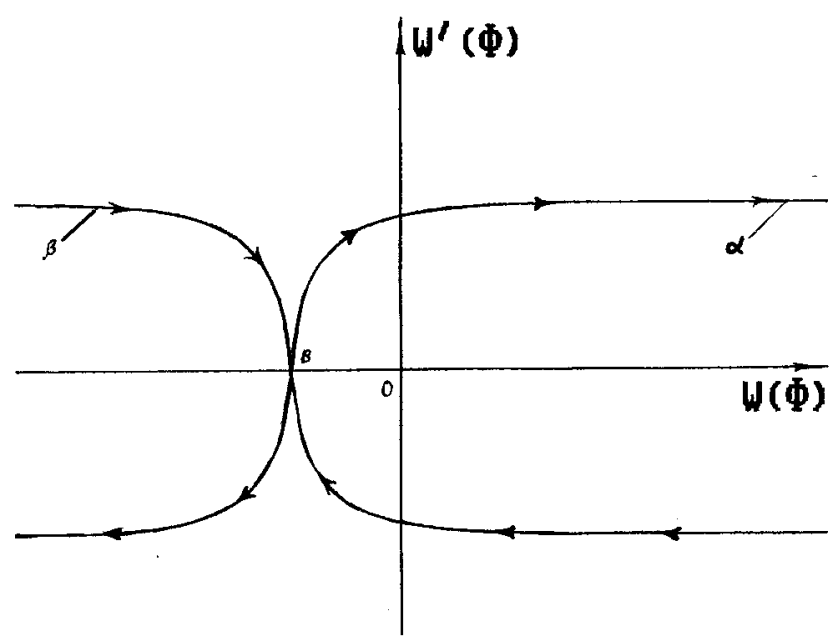

Fig. 4. The phase place $\left(\mathbf{W}, \mathbf{W}^{\prime}\right)$ of the solitary waves. Curves correspond to the relation (35). The constant $\mathbf{B}=-1 ;-1<\mathbf{W}<4$ (branch $\alpha),-4<\mathbf{W}<-1$ (branch $\beta$ ). 
A period of the vibro-impact motion is a time of the passage from $A_{1}$ to $A_{2}$ along the phase trajectory. We consider a period of the vibro-impact motion by branch $\alpha$,

$$
\mathbf{T}^{(\alpha)}=\frac{1}{V \epsilon} \ln \frac{\mathrm{e}^{(L-\mathbf{B} \epsilon) V}+\sqrt{\mathrm{e}^{2(L-\mathbf{B} \epsilon) V}-1}}{\mathrm{e}^{(L-\mathbf{B} \epsilon) V}-\sqrt{\mathrm{e}^{2(L-\mathbf{B} \epsilon) V}-1}} \quad \text { or } \mathbf{T}^{(\alpha)}=\frac{2}{V \epsilon} \ln \left(\mathrm{e}^{(L-\mathbf{B} \epsilon) V}+\sqrt{\mathrm{e}^{2(L-\mathbf{B} \epsilon) V}-1}\right) .
$$

One obtains from (38)

$$
\begin{aligned}
& \mathbf{T}_{\min }^{(\alpha)}=0 \quad \text { if } \mathbf{B}=L / \epsilon ; \\
& \mathbf{T}_{\max }^{(\alpha)}=\frac{2}{V \epsilon} \ln \left(\mathrm{e}^{2 L V}+\sqrt{\mathrm{e}^{4 L V}-1}\right) \text { if } \mathbf{B}=-L / \epsilon .
\end{aligned}
$$

Consider the branch $\beta$. One has:

$$
\Phi=\frac{1}{V \epsilon} \ln \left(\mathrm{e}^{(L+\mathbf{B} \epsilon) V} \pm \sqrt{\mathrm{e}^{2(L+\mathbf{B} \epsilon) V}-1}\right) .
$$

A period of the motion by branch $\beta$ is a time of the passage from $B_{2}$ to $B_{1}$ :

$$
\begin{aligned}
& \mathbf{T}^{(\beta)}=\frac{2}{V \epsilon} \ln \left(\mathrm{e}^{(L+\mathbf{B} \epsilon) V}+\sqrt{\mathrm{e}^{2(L+\mathbf{B} \epsilon) V}-1}\right) \quad \text { and } \quad \mathbf{T}_{\min }^{(\beta)}=0 \quad \text { if } \mathbf{B}=-L / \epsilon, \\
& \mathbf{T}_{\max }^{(\beta)}=\frac{2}{V \epsilon} \ln \left(\mathrm{e}^{2 L V}+\sqrt{\mathrm{e}^{4 L V}-1}\right) \quad \text { if } \mathbf{B}=L / \epsilon .
\end{aligned}
$$

Consequently, the minimal and maximal times of the motion between barriers are not dependent on the vibration modes, the times are the functions of the system parameters only.

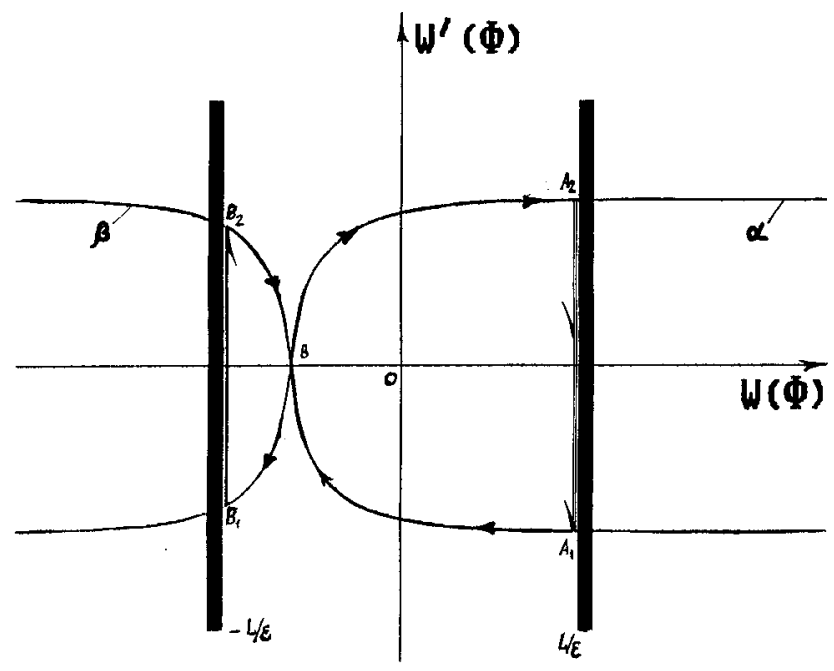

Fig. 5. Phase portrait of the solution (34) with impact, the variant 1 . The constant $\mathbf{B}=-1, L / \epsilon=1.7,-1<\mathbf{W}<4$ (branch $\alpha$ ), -4 $<\mathbf{W}<-1$ (branch $\beta$ ). Point $A_{1}$ corresponds to the sign ' - ' in relation (37), here $\Phi=\Phi_{1}$; point $A_{2}$ corresponds to the sign ' + ' in (37), here $\Phi=\Phi_{2} ; \Phi_{1}=-\Phi_{2}$. 


\subsection{Consider the variant 2}

The corresponding phase portrait is presented in Fig. 6 . Here $\mathbf{B}=-2, L / \epsilon=1.7,-2<\mathbf{W}<6$. It is only presented in branch $\alpha$.

$T_{1}$ is a time of the motion from $A_{1}$ to $B_{1} ; T_{2}$ is the same from $B_{2}$ to $A_{2}$. Let $\mathbf{B}=-L / \epsilon-\mathbf{G}(\mathbf{G}>0)$.

One has $\mathbf{W}=L / \epsilon$ if

$$
\mathbf{W}=L / \epsilon \quad \text { if } \quad \Phi=\frac{1}{V \epsilon} \ln \left(\mathrm{e}^{(2 L+\mathbf{G} \epsilon) V} \pm \sqrt{\mathrm{e}^{2(2 L+\mathbf{G} \epsilon) V}-1}\right)
$$

and

$$
\mathbf{W}=-L / \epsilon \quad \text { if } \Phi=\frac{1}{V \epsilon} \ln \left(\mathrm{e}^{\mathbf{G} \epsilon V} \pm \sqrt{\mathrm{e}^{2 \mathbf{G} \epsilon V}-1}\right) .
$$

One obtains from $(41)$

$$
\begin{aligned}
& T_{1}=\frac{1}{V \epsilon} \ln \frac{1-\sqrt{1-\mathrm{e}^{-2 \mathbf{G} \epsilon V}}}{\mathrm{e}^{2 L V}-\sqrt{\mathrm{e}^{4 L V}-\mathrm{e}^{-2 \mathbf{G} \epsilon V}}} ; \\
& T_{2}=\frac{1}{V \epsilon} \ln \frac{\mathrm{e}^{2 L V}+\sqrt{\mathrm{e}^{4 L V}-\mathrm{e}^{-2 \mathbf{G} \epsilon V}}}{1+\sqrt{1-\mathrm{e}^{-2 \mathbf{G} \epsilon V}}} .
\end{aligned}
$$

It is possible to make sure of $T_{1}=T_{2}$. Consequently,

$$
\mathbf{T}^{(\alpha)}=T_{1}+T_{2}=\frac{2}{V \epsilon} \ln \frac{\mathrm{e}^{2 L V}+\sqrt{\mathrm{e}^{4 L V}-\mathrm{e}^{-2 \mathbf{G} \epsilon V}}}{1+\sqrt{1-\mathrm{e}^{-2 \mathbf{G} \epsilon V}}}
$$

and

$$
\mathbf{T}_{\max }^{(\alpha)}=\frac{2}{V \epsilon} \ln \left(\mathrm{e}^{2 L V}+\sqrt{\mathrm{e}^{4 L V}-1}\right)=\mathbf{T}_{\max } \quad \text { if } \mathbf{G}=0
$$

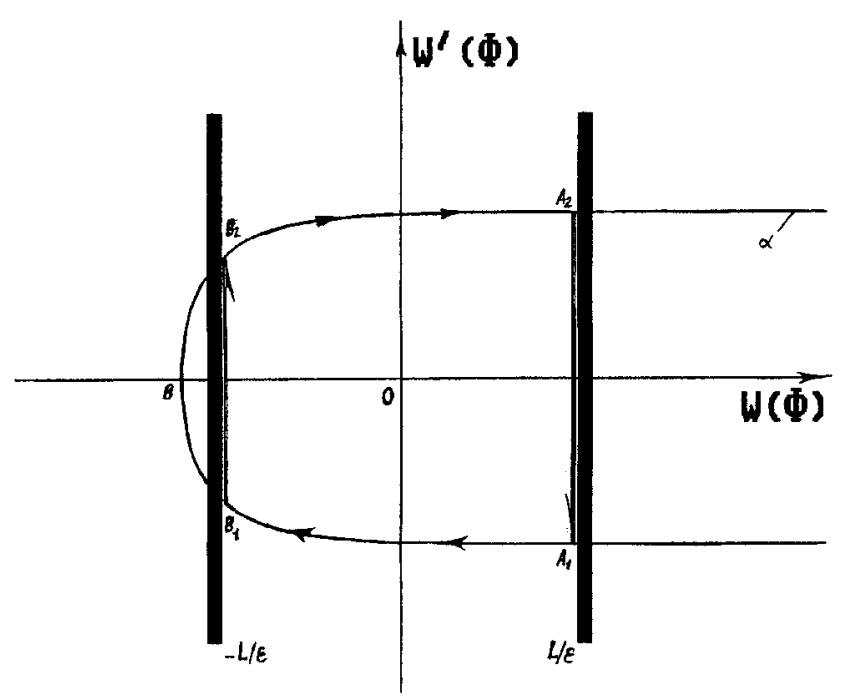

Fig. 6. Phase portrait of the solution (34) with impact, the variant 2 . The constant $\mathbf{B}=-2, L / \epsilon=1.7,-2<\mathbf{W}<6$. It is only presented the branch $\alpha$. Point $A_{1}$ corresponds to the sign ' - ' in relation (37), here $\Phi=\Phi_{1}$; point $A_{2}$ corresponds to the sign ' + ' in (37), here $\Phi=\Phi_{2} ; \Phi_{1}=-\Phi_{2}$. 
By analogy with the results one obtains $(\mathbf{B}=L / \epsilon+\mathbf{G} ; \mathbf{G}>0)$

$$
\begin{aligned}
& \mathbf{T}^{(\beta)}=\frac{2}{V \epsilon} \ln \frac{\mathrm{e}^{2 L V}+\sqrt{\mathrm{e}^{4 L V}-\mathrm{e}^{-2 \mathbf{G} \epsilon V}}}{1+\sqrt{1-\mathrm{e}^{-2 \mathbf{G} \epsilon V}}} \\
& \mathbf{T}_{\max }^{(\beta)}=\frac{2}{V \epsilon} \ln \left(\mathrm{e}^{2 L V}+\sqrt{\mathrm{e}^{4 L V}-1}\right)=\mathbf{T}_{\max } \quad \text { if } \mathbf{G}=0 .
\end{aligned}
$$

It is possible to make sure that $\mathbf{T}^{(\alpha)}=\mathbf{T}^{(\beta)}$ (if the constants $\mathbf{G}$ are coinciding).

\subsection{Consider the variant 3}

The corresponding situation is depicted in Fig. 7. Here $\mathbf{B}=-1, L / \epsilon=1,-1<\mathbf{W}<4$ (branch $\alpha$ ), $-4<\mathbf{W}<-1$ (branch $\beta$ ).

The variant can be represented as a special case of the variant 1 .

In this case: $\mathbf{T}^{(\alpha)}=\mathbf{T}_{\max }, \mathbf{T}^{(\beta)}=\mathbf{T}_{\min }=0$.

Simultaneously the variant 3 can be represented as a special case of the variant 2 .

In this case branch $\beta$ is absent and $\mathbf{T}^{(\beta)}=0, \mathbf{T}^{(\alpha)}=\mathbf{T}_{\max }$.

\section{Construction of periodical vibro-impact solutions}

As is obvious from the preceding, there are jumps of the function $\mathbf{W}^{\prime}(\Phi)$ at the point of impact. The function $\mathbf{W}^{\prime \prime}(\Phi)$ is singular. Rejected inertia terms containing $\mathbf{U}^{\prime \prime}$ are not small. Therefore, the examined vibro-impact approximation circumscribes sufficiently well only for the transverse displacements $\mathbf{W}(\Phi)$.

Consider, in the first place, the variant 1 (Section 5.1). There are impacts to single barriers in this case. The phase values corresponding to impact times are the next: $\Phi=\Phi_{1}$ and $\Phi=\Phi_{2}$. In the chosen coordinate system $\Phi_{1}=-\Phi_{2}$ and $T=\Phi_{1}-\Phi_{2}$ is a time of the motion between impacts [see (38); $\Phi_{1}<0$, $\left.\Phi_{2}>0\right]$.

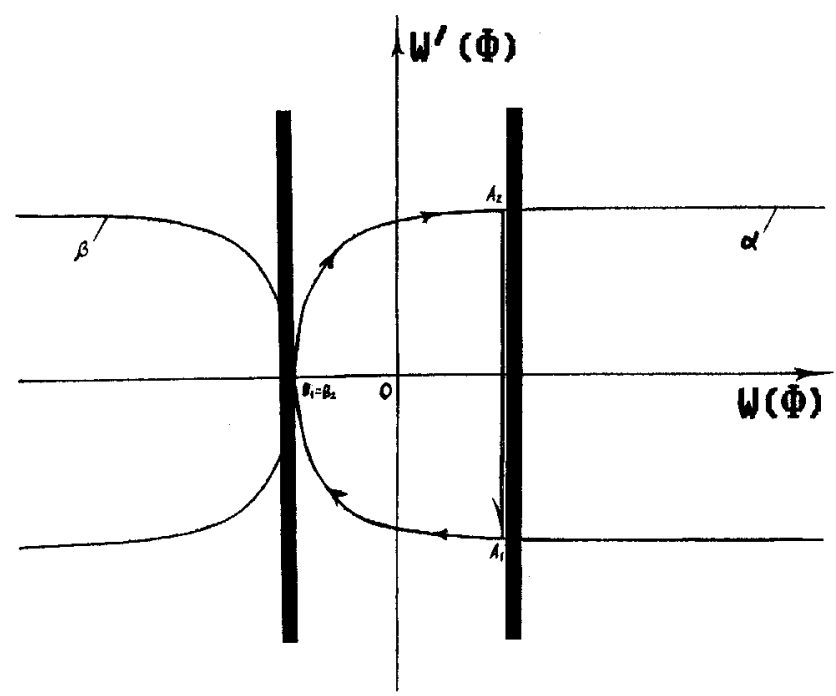

Fig. 7. Phase portrait of the solution (34) with impact, the variant 3 . The constant $\mathbf{B}=-1, L / \epsilon=1,-1<\mathbf{W}<4$ (branch $\alpha$ ), $-4<$ $\mathbf{W}<-1$ (branch $\beta$ ). Point $A_{1}$ corresponds to the sign ' - ' in relation (37), here $\Phi=\Phi_{1}$; point $A_{2}$ corresponds to the sign ' + ' in (37), here $\Phi=\Phi_{2} ; \Phi_{1}=-\Phi_{2}$. 
The vibro-impact discontinuous solution under consideration can be expressed as a continuous periodical function. Namely, a special non-smooth transformation can be introduced:

$$
\xi(\Phi)=T / 2+\{\Phi / T\} T
$$

where curly brackets mean a selecting of the fractional component. The transformation (44) is like the non-smooth saw-tooth time transformation by Pilipchuk (1996) for essentially non-linear systems close to the vibro-impact ones and to the non-smooth transformation by Zhuravlev (1977).

One has $-T / 2 \leqslant \xi(\Phi) \leqslant T / 2$ if $-\infty<\Phi<+\infty$. Impact points are the following:

$$
\Phi=T / 2+T k ; \quad k=0, \pm 1, \pm 2, \ldots
$$

A solution of the problem is a function $\mathbf{W}(\xi(\Phi))$ defined on $-\infty<\Phi<+\infty$. The functions $\mathbf{W}(\xi(\Phi))$ and $\mathbf{W}^{\prime}(\xi(\Phi))$ are presented in Fig. 8 on the interval $[-T / 2,3 T / 2]$.

Consider present the variant 2 (Section 5.2). In this case there are impacts to both barriers.

The impacts in the vicinity of the origin takes place in the following points (Fig. 6):

$A_{1}$ : here $\Phi_{1}<0$, coordinates of the barriers: $\Phi=L / \epsilon($ branch $\alpha)$, and $\Phi=-L / \epsilon($ branch $\beta)$;

$B_{1}$ : here $\Phi_{2}<0$, coordinates of the barriers: $\Phi=-L / \epsilon$ (branch $\left.\alpha\right)$, and $\Phi=L / \epsilon$ (branch $\left.\beta\right)$;

$B_{2}$ : here $\Phi_{3}>0$, coordinates of the barriers: $\Phi=-L / \epsilon($ branch $\alpha)$, and $\Phi=L / \epsilon$ (branch $\beta$ );

$A_{2}$ : here $\Phi_{4}>0$, coordinates of the barriers: $\Phi=L / \epsilon$ (branch $\left.\alpha\right)$, and $\Phi=-L / \epsilon$ (branch $\beta$ ).

In the chosen coordinate system $\Phi_{1}=-\Phi_{4}, \Phi_{2}=-\Phi_{3} ; T=\left(\Phi_{2}-\Phi_{1}\right)+\left(\Phi_{4}-\Phi_{3}\right)$ [see (41)-(43)].

The transformation

$$
\xi(\Phi)=\left\{\begin{array}{l}
\Phi_{1}+\{\Psi /(T / 2)\} T / 2 \text { if } \Psi \leftarrow T / 2 \\
\Phi_{3}+\{(\Psi-(T / 2)) /(T / 2)\} T / 2 \text { if } \Psi \geq T / 2
\end{array}\right.
$$

where $\Psi=\{\Phi / T\} T$, gives us the periodical representation of the vibro-impact motion.

Impact points are the following:

$$
\Phi=\Phi_{1}+(T / 2) 2 k ; \quad k=0, \pm 1 \pm 2, \ldots
$$

and

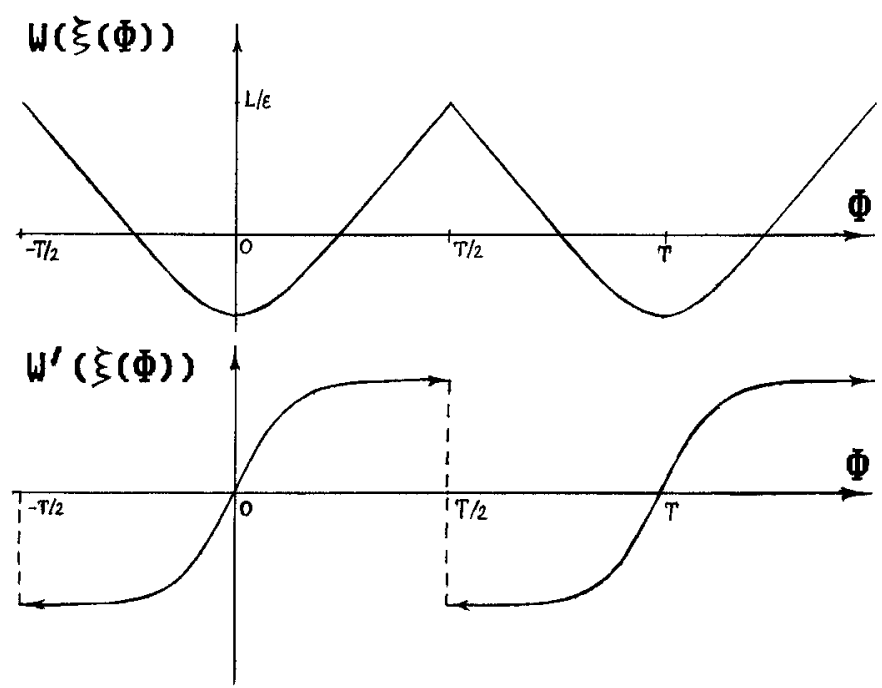

Fig. 8. Diagram of the functions $\mathbf{W}(\xi(\Phi))$ and $\mathbf{W}^{\prime}(\xi(\Phi))$ on the interval $[-T / 2,3 T / 2]$ in the case of impacts to a single barrier. Here the variable $\xi(\Phi)$ is given by formula (44). 


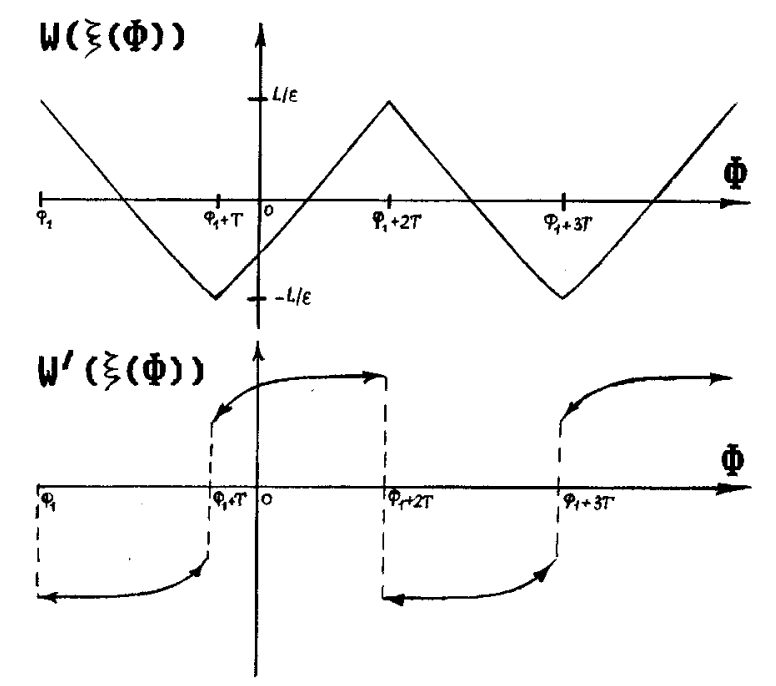

Fig. 9. Diagram of the functions $\mathbf{W}(\xi(\Phi))$ and $\mathbf{W}^{\prime}(\xi(\Phi))$ on the interval $\left[\Phi_{1}, \Phi_{1}+4 T\right]$ in the case of impacts to both barriers. Here the variable $\xi(\Phi)$ is given by formula $(45)$.

$$
\Phi=\Phi_{1}+(T / 2)(2 k+1) ; \quad k=0, \pm 1, \pm 2, \ldots,\left(\Phi_{2}=\Phi_{1}+T / 2\right) .
$$

A solution of the problem is a function $\mathbf{W}(\xi(\Phi))$ defined on $-\infty<\Phi<+\infty$. The functions $\mathbf{W}(\xi(\Phi))$ and $\mathbf{W}^{\prime}(\xi(\Phi))$ are presented in Fig. 9 on the interval $\left[\Phi_{1}, \Phi_{1}+4 T\right]$ (here $\left.\Phi_{1}<0\right)$.

\section{Conclusions}

We analyzed transversal traveling waves in one-dimensional essentially non-linear infinite elastic systems: chains and beams. In the framework of a long wavelength approximation the result was that the non-linear systems with regard to impact (only the case of absolutely elastic impact is regarded) sometimes assume the solitary traveling waves. An analysis of the phase planes of a long wavelength traveling wave gives us conditions of the solitary waves existence. A special non-smooth transformation which gives us a periodical representation of the vibro-impact motion was also introduced. The presented analysis can be extended to other non-linear elastic systems including two-dimensional, such as plates or membranes.

\section{Acknowledgements}

The authors would like to thank Professors I. V. Andrianov and V. Z. Gristchak for useful discussions.

\section{References}

Clarkson, P.A., Leveque, R.J., Saxton, R., 1986. Solitary wave interactions in elastic rods. Stud. Appl. Math. 75, $95-122$.

Emaci, E., Nayfeh, T.A., Vakakis, A.F., 1997. Numerical and experimental study of non-linear localization in a flexible structure with vibro-impact. ZAMM 77 (7), 527-541.

Farahanchi, F., Shaw, S.W., 1994. Chaotic and periodic dynamics of a slider-crank mechanisms with slider clearance. Journal of Sound and Vibration 177 (3), 307-324.

Goldsmith, W., 1960. Impact. The Theory and Physical Behaviour of Colliding Solids. Arnold, London. 
Han, R.P.S., Luo, A.C.J., 1995. Chaotic motion of a horizontal impact pair. Journal of Sound and Vibration 181 (2), 231-250.

Ivanov, A.P., 1993. Analytical methods in the theory of vibroimpact systems. Prikl. Mat. Mech. (PMM, USSR) 57 (2), $5-21$.

Ivanov, A.P., 1994. Impact oscillations: linear theory of stability and bifurcations. Journal of Sound and Vibration 178 (3), $361-$ 378.

Ivanov, A.P., 1995. About the multiple impact. Prikl. Mat. Mech (PMM, USSR) 59 (6), 930-946.

Kauderer, H., 1958. Nichtlinear Mechanic. Springer Verlag, Berlin.

Kosevich, A.M., Kovalev, A.S., 1975. Self-localization of vibrations in a one-dimensional anharmonic chain. Sov. Phys.- JETP 40 (5), 891-896.

Manevitch, L.I., Mikhlin, Yu V., Pilipchuk, V.N., 1989. The Method of Normal Vibrations for Essentially Non-linear Systems. Nauka, Moscow (in Russian).

Masri, S.F., Caughey, T.K., 1966. On the stability of the impact damper. J. Appl. Mech. 33, 582-592.

Mikhlin, Yu V., 1996. Normal vibrations of a general class of conservative oscillators. Nonlinear Dynamics 11, 1-15.

Mikhlin, Y.V., Vakakis, A.F., Salenger, G., 1998. Direct and inverse problems encountered in vibro-impact oscillations of a discrete system. Journal of Sound and Vibration 216 (2), 227-250.

Moon, F., Holmes, W., Knoury, S., 1991. Symbol dynamic maps of spatial-temporal chaotic vibrations in a string of impact oscillatiors. Chaos 1 (1), 65-68.

Nagaev, R.F., 1985. Mechanical Processes with Repeated Damped Impacts. Nauka, Moscow (in Russian).

Ostrovsky, L.A., Sutin, A.M., 1977. Non-linear elastic waves in rods. Prikl. Mat. Mech. (PMM, USSR) 41 (3), $531-537$.

Pilipchuk, V.N., 1996. Analytical study of vibrating systems with strong non-linearities by employing saw-tooth time transformations. Journal of Sound and Vibration 192 (1), 43-64.

Potapov, A.I., 1985. Non-linear Strain Waves in Beams and Plates. Gorky University, Gorky (in Russian).

Samsonov, A.M., 1984. Soliton evolution in a rod with variable cross section. Sov. Physics-Doklady 29 (7), $586-587$.

Samsonov, A.M., 1995. Non-linear strain waves in elastic wave-guides. Int. Centre for Mech. Sciences. Courses and Lectures No. 341. Springer-Verlag, Wien, New York.

Shaw, S.W., 1985. The dynamics of a harmonically excited systems having rigid amplitude constraints. Part I: subharmonic motions and local bifurcations, and Part II: chaotic motions and global bifurcations. Journal of Applied Mechanics 52, 453464.

Shaw, S.W., Holmes, P.J., 1983. Periodically forced linear oscillator with impact-chaos and long term motions. Phys. Rev. Lett. 51, 623-626.

Shaw, J., Shaw, S.W., 1989. The onset of chaos in a two-degree-of-freedom impacting system. J. Appl. Mech. 56, $168-174$.

Soerensen, M.P., Christiansen, P.L., Lomdahe, P.S., 1984. Solitary waves in non-linear elastic rods, I. J. Acoust. Soc. Amer. 76, $871-879$

Soerensen, M.P., Christiansen, P.L., Lomdahe, P.S., Skovgaard, O., 1987. Solitary waves in non-linear elastic rods, II. J. Acoust. Soc. Amer. 81 (6), 1718-1722.

Toda, M., 1981. Theory of Non-linear Lattices. Springer Verlag, Berlin, New York.

Vakakis, A.F., Manevitch, L.I., Mikhlin, Yu V., Pilipchuk, V.N., Zevin, A.A., 1996. Normal Modes and Localization in Nonlinear Systems. John Wiley, New York.

Valkering, T.P., 1994. Non-trivial dynamics in a driven string with impact nonlinearity. Journal of Sound and Vibration 175 (5), $397-422$.

Zhuravlev, V.F., 1977. Investigation of certain vibration-impact systems by the method of non-smooth transformation. Izv. AN SSSR, Mechanika Tverdogo Tela. 12 (6), 24-28. 